\title{
Colour Coding Navigation: "Triage" Techniques to Improve Compliance in Breast Cancer Patients Requiring Primary Chemotherapy
}

Carol Ann Benn (1), Yastira Ramdas (), Barend van den Bergh (1), Naa-Lamle Bannerman (), Dominic van Loggerenberg (1), Tia van Loggerenberg (i), Vernon Shaw

Netcare Breastcare Centre of Excellence, Milpark Hospital, Johannesburg, South Africa

\begin{abstract}
Objective: This is a pilot study to assess whether a file-colour-coded triage navigation system for patients on primary chemotherapy improves compliance and adherence and if it decreases defaulting.

Materials and Methods: All breast cancer patients are discussed in a multidisciplinary meeting. All patients are triaged before starting on primary chemotherapy based on their specific challenges and beliefs and are consulted by the navigation team and contacted before the beginning of treatment and after each chemotherapy session by a navigator in the unit. File stratification for ease of navigation was instituted by a colour code dot into three groups. The three groups are:

Code Green: Compliant on treatment

Code Yellow: Side effects on treatment/ considering defaulting

Code Red: Non-compliant

The code red patients were further assessed in terms of reasons for non-adherence or non-compliance:

Fear of chemotherapy side effects

The belief that chemotherapy kills the patient

Interest in "alternative treatment regimens"

Other barriers to treatment as identified by the navigators

Results: The system allows the navigation team to focus on which patients require specific navigation and inform the treating oncologists. Code green patients were courtesy called after each chemotherapy session. The code yellow patients had early involvement with the survivorship team to ensure appropriate management of any side effects. Access to the complimentary oncology navigator and complementary health website was instituted. The oncology navigator visited each patient at the oncology unit on the day the patient was due to have chemotherapy. For Code red 1 and 2, a "buddies" network of patients who have been through similar treatment regimens was assigned by the navigation team. This was coordinated by patient navigators (trained counsellors who have had breast cancer treatment). Code red three was managed by a complementary health specialist who understood the value of chemotherapy. For Code red 4, the oncology navigator manages the concerns from finances services to family issues. For the 122 patients in total for primary chemotherapy, stratification was as follows:

Code Green $=64.8 \%$

Code Yellow $=27.0 \%$

Code Red $=8.2 \%$.

Conclusion: This system provides the Multidisciplinary team with the opportunity to improve patient adherence/compliance with primary chemotherapy. $80 \%$ of the code red patients eventually agreed to receive the recommended treatment. Navigation enhanced patient supervision, and the coding system improved patient primary chemotherapy adherence. Such a system would benefit larger oncological practices to improve primary chemotherapy adherence by empowering the navigation team to identify patients requiring more intensive navigation supervision.
\end{abstract}

Keywords: Breast cancer, navigation, neoadjuvant chemotherapy, patient compliance, patient adherence

Cite this article as: Benn CA, Ramdas Y, van den Bergh B, Bannerman NL, van Loggerenberg D, van Loggerenberg T, Shaw V. Colour Coding Navigation: "Triage" Techniques to Improve Compliance in Breast Cancer Patients Requiring Primary Chemotherapy. Eur J Breast Health 2020; 16(4): $262-266$.

\section{Introduction}

\section{Understanding the geography}

The Netcare Milpark Breast Care Centre is a multidisciplinary breast unit established in 2000 and sees patients that are 'private' or funded by medical aid. The unit is based in Johannesburg, South Africa. Since its formation, it has seen over 24000 new patients 
and coordinated the diagnosis and management of over 10000 breast cancer patients. The unit treated 488 newly diagnosed breast cancer patients in 2017, with: 122 undergoing primary chemotherapy; 42 undergoing primary endocrine therapy (5 of whom are now deceased); 43 presented with or progressed to metastatic disease; 253 underwent primary surgery (2017 approved data). The unit has had full NAPBC accreditation since 2016 (November). Patient demographics include women from all over South Africa, as well as from other African countries, resulting in many challenges with regards to navigation. The Breast Care Centre has a sister unit that attends to patients that are not funded by medical aid (insurance), at Helen Joseph hospital. Both units are managed similarly, and headed up by the same specialist.

Premature termination of chemotherapy is linked to higher mortality rates which are particularly prevalent with women in low socio-economic environments-sub-Saharan health care systems battle with the ever-increasing breast cancer treatment requirements. The unintended consequence is clinicians spending less time attending to the patient's psychological states, thus leading to inefficient chemotherapy adherence. In order to improve primary chemotherapy adherence, three primary navigators are assigned to track/monitor patients and give feedback of results to the multidisciplinary team. The system was implemented through triaging primary chemotherapy patients and assigning each a colour code. The primary purpose of this study was to aid the navigation team in tracking patients on primary chemotherapy thus improving oversight and management. The aim was not to study the many variables that contribute to non-adherence and poor compliance but rather to identify those patients requiring a more intensive navigation program.

\section{Background}

Breast cancer is the most common cancer diagnosed in South African women, accounting for $21.78 \%$ of cancers diagnosed in 2014 (South African National Cancer Registry) (1). Breast cancer was also found to be the most common cause of cancer death in 11 regions of the world (2). The relatively higher mortality rate of breast cancers diagnosed in countries with no radiology screening programs makes its diagnosis too daunting and traumatic for all patients. Fortunately, treatment modalities are continually progressing, allowing clinicians, the opportunity to improve survival outcomes and delay disease progression. Longstanding evidence supports the use of chemotherapy, with or without target therapies for many biological types of breast cancer, with or without nodal disease, with a resultant good response to a variety of chemotherapy agents (3). Despite the benefits of chemotherapy being established in the literature, being advised that one should start with chemotherapy as primary treatment is often met with opposition from

\section{Key Points}

- Triage techniques such as this are useful tools in assisting oncology navigators in refining and tailoring their approach to the navigation of a patient during their treatment.

- Understanding the contributing factors behind a patient's assigned Colour Code provides the navigator with improved background knowledge of a patient's experiences, allowing for improved resolution to such factors.

- The implementation and management of a 'buddies network' allows for patient - patient interaction and support, allowing for more interpersonal support being offered to patients experiencing anxiety or presenting with concerns on their treatment. many patients. In the current technological era, it is commonplace for patients to explore their diagnoses online where they are met with a host of different opinions on available treatment modalities. The patient's decision, pertaining to their treatment choice, is formed by an array of factors, including psychological and social, and is categorically not based purely on scientific data.

Breast cancer, in recent times, is treated using a more personalized approach based on the different biological subtypes of the disease (4). The concept of determining treatment based on the biology of breast cancer in addition to the stage is a difficult concept for many patients to grasp and reasons as to why specific treatment modalities may be preferred over others can be confusing for the patient if not properly communicated. Additionally, complementary and alternative medicine (CAM) has grown in popularity over the years (5), with more patients investigating this route for the treatment of cancer as a way to avoid the presumed and actual side effects of conventional treatment. When investigating reasons for CAM use in cancer patients, Paltiel et al. found significant associations between CAM use and attending supportive psychotherapy, unmet needs, helplessness, and worse emotional and social function (6). These findings suggest that psycho-social needs to play a significant role in oncological treatment and more attention must be placed on the patient's psychology/ideologies around cancer in order to improve treatment outcomes.

While some cancer patients prefer for their physician to have complete control over treatment choices, it has been found that the majority prefer shared decision making (7). The word 'compliance' suggests that the patient incontrovertibly follows the doctor's recommendations, while adherence infers that the patient is not forced to comply to a specific treatment and is instead part of an allied effort to determine the best treatment option for their case (8). It also implies that the patient can not solely be held responsible for non-adherence and that it is the responsibility of both the patient and health provider to put in place frameworks to support the decided treatment. Blind compliance may have sufficed in times when a vertical doctor-patient relationship was the norm. However, in recent times where medical information is more widespread, patients are more involved in their management. When a patient is intricately involved in the decision-making process, they are more likely to adhere to a specific treatment long term. Discussions pertaining to treatment must integrate the views of the health professional, allied medical practitioners and the patient. Lack of consultation with the patient on their views of the proposed treatment prevents the practitioner from identifying potential barriers to adherence and, on the other hand, early involvement of the patient in their treatment decision-making process assists in curbing impending nonadherence or non-compliance.

Navigation interventions have been frequently applied in breast cancer screening and early diagnosis. However, they have not commonly been implemented to address adherence to treatment (9). Systems must be put in place to ensure the patient's participation in treatment decision making and their continued adherence to the decided regimen. A navigation system where barriers to treatment can be promptly ascertained and addressed has the potential to decrease rates of non-adherence and improve patient outcomes. Stratification of patient files is not new. By using a colour-coded system, such as that used in trauma triage, the oncologist and oncology navigator can dedicate more time and resources with those patients who are either battling with the concept of primary chemotherapy; struggling with side effects; have a fundamentally anti- 
chemotherapy ideology, or logistic and financial issues preventing treatment adherence.

The trauma triage concept of red, yellow, and green is based on the area of disaster medicine. It is particularly useful as dividing into three helps with lessening patient load in order to treat the required patients with limited resources efficiently. A cancer diagnosis can seem, to the patient at least, as akin to a personal disaster scenario. The reality of increased numbers of patients being treated for breast cancer, coupled with the reality of resource disparity requires a system where optimum use of the available resources can be continuously developed and updated.

Altering this concept to a file-coding system in order to determine potential patient nonadherence/non-compliance to chemotherapy, particularly in the neoadjuvant setting, is invaluable. Most patients are reticent for many reasons to start and adhere with recommended chemotherapy regimens. This is further exacerbated by the fact that after being diagnosed by a radiologist; surgical oncologists explain the treatment routine; then further discussed in multidisciplinary meetings before final referral to medical oncology units for the commencement of chemotherapy treatment. This assembly of cross medical discipline interaction requires substantial navigation. Patient inclusion can be variable in this scenario, and specific systems must be put in place to safeguard against the loss of contact with patients, and ultimately, non-adherence.

\section{Study aim}

To assess the benefit of implementing a colour coded navigation system for the early identification, and appropriate management, of non-adherence and/or non-compliance to primary chemotherapy.

\section{Scope}

A pilot study conducted in the Netcare Breast Care Centre of Excellence (BCCE), a single unit in Johannesburg South Africa that has been operational and running as a multidisciplinary breast care centre since 2000 . The unit sees approximately 450 newly diagnosed breast cancer patients a year.

\section{Materials and Methods}

\section{Ethics committee approval specifics}

No ethics committee approval was garnered nor required for this study as no additional information other than standard unit process was gathered on patients. The materials used were standard internal medical files. The methodology implemented involved a coloured sticker on a file to streamline and organise navigation in the unit as opposed to acquiring information directly from patients. All patient files accessed had signed consent forms signed by the patients when they first attended the centre. The ethics covering the use of this information is governed by our MIDAS Protocol. Below are the reference numbers as approved by the parent hospital of the unit and the ethics committee that approved the protocol.

Netcare Trial Number: TRIAL-2017-0035

PharmaEthics Ref No: 170416525

\section{Study design}

A retrospective qualitative observational pilot study of patients who were assigned colour coding as part of a navigation system

\section{Patient selection}

All patients seen in 2017, whose management plan included primary chemotherapy, were eligible for inclusion to the trial. All newly diagnosed breast cancer patients are discussed in a multidisciplinary meeting before starting treatment arms. According to 2017 approved audited data, a total of 122 patients who underwent primary chemotherapy in 2017 were selected. Patients starting primary chemotherapy in other units that moved to our centre were excluded. Patients who had surgery elsewhere were also excluded.

\section{Colour coding navigation protocol}

A navigator contacted all patients undergoing primary chemotherapy after each chemotherapy session this was standard unit navigation protocol. All patients are triaged before starting on primary chemotherapy based on their specific challenges and beliefs and are consulted by the navigation team and contacted before the beginning of treatment and after each chemotherapy session by a navigator in the unit. Feedback from the patient was used to assign each patient a colour code based on their reported response to being planned for chemotherapy. Code green was assigned to patients that were not against NACT and were adherent with no side effects. Code yellow was assigned to patients that had some reservations and were experiencing side effects and having issues with adherence. Code Red was the designation for patients that were non adherent or refusing treatment and who were against beginning treatment. These patients were further assessed in terms of reasons for non-adherence. The reasons for non-adherence or refusal of treatment (code red) were grouped into the following categories:

Anxiety - fear of chemotherapy side effects, including those experiencing significant side effects to chemotherapy (this was not quantified).

Psychological - the belief that chemotherapy "kills the patient" with absolute refusal to partake in the treatment.

Alternative - preference for alternative/homoeopathic treatment regimens.

Social - barriers to treatment ranging from financial to logistical. The navigator identified these reasons.

The code green patients received a courtesy call after each chemotherapy session. The code yellow patients were managed by early involvement of the unit's survivorship/navigation team to ensure careful management of side effects with the addition of the complementary health navigation specialist in the unit to explain benefits and harms of alternative medicines. All these patients were met at the chemo unit on the morning of their chemo by the oncology navigator to ensure that anxiety around the chemo was managed. Code Red 1 and 2 category patients were managed by a buddy system of patients who have been through similar treatment regimes in coordination with the navigation team. This 'buddy' system falls under the umbrella of the Breast Health Foundation and is managed by the patient advocate on the BPLC (breast program leadership committee) who is the head of the Breast Health Foundation. Code red three was managed by a complementary health specialist who understands the value of chemotherapy yet has background training in complementary medicine. The patient navigation team managed code-red four patients. The latter refer patients to the appropriate services in the community (i.e. social worker, financial aid or psycho oncology) or within the health system to aid with logistics/family responsibility and financial reasons for potential non-adherence to oncology treatment. 


\section{Study endpoints}

To assess whether the colour coded navigation system for primary chemotherapy improves adherence/compliance to treatment regimens. To determine whether a roll-out and prospective study across both units were feasible to develop intelligent systems for the future to improve patient care in low resource environment settings (10).

To determine attitudes, beliefs, social circumstances, and other factors that pose a barrier to adherence/compliance to primary chemotherapy in breast cancer patients. This secondary endpoint was to determine the feasibility of a masters study in cross-cultural navigation to be performed by an oncology navigator who spoke all 11 languages and had an interest in both cultural beliefs and cancer.

\section{Results}

This system provided the MDM team, via the navigator, with the opportunity to improve patient adherence on primary chemotherapy. The allocation of the colour coding was based on a navigator assessment of patient reticence or compliance with the prescribed treatment regimen, and the specific breakdown of the stratification can be seen in Table 1. This is part of a pilot concept for a navigation doctorate. $80 \%$ of the code red patients, eight, eventually agreed to recommended treatment. The system allows the navigation team to focus on which patients require specific and intensive navigation and then to coordinate with the oncologists, thus improving adherence to treatment regimes. All Code Yellow patients completed their chemotherapy regimens during the course of the study, as did all Code Green patients.

The sub categorization of Code Red patients can be seen in Table 2 , whereby one can see that $40 \%$ of patients harbored interests in alternative medicine and therapies as opposed to receiving chemotherapy, $20 \%$ feared the possible side effects of taking chemotherapy while only $10 \%$ (the smallest of the group) believed that chemotherapy would kill them. The final 30\% of Code Red patients were identified to have a range of barriers that contributed to their noncompliance as determined by the navigators. This included geographical barriers and differences in family opinions amongst others.

\begin{tabular}{|lcc|}
\hline \multicolumn{2}{l}{ Table 1. Patient stratification } \\
\hline Code & Description & No patients, N \\
\hline Red & Non-adherent or non-compliant & 10 \\
Yellow & Reserved opinion & 33 \\
Green & Adherent and Compliant & 79 \\
\hline & Total & 122 \\
\hline
\end{tabular}

Table 2. Code Red sub classification categorisation

\begin{tabular}{lc} 
Category & \# people \\
\hline 1 & 2 \\
2 & 1 \\
3 & 4 \\
4 & 3
\end{tabular}

\section{Discussion and Conclusion}

Understanding the complex issues around patient adherence/compliance requires a multi-factorial approach not just to treatment options but to understanding the intricacies of why patients choose adherence over non-adherence to chemotherapy. The value of multidisciplinary medicine is not just in the concept of discussing different options in patient management in a medical cross-specialty team environment, but also that of learning from the members of different specialties. This concept should be extended past that of specialists treating oncology patients to learning from other disciplines. The field of trauma medicine has taught medical specialists the value of a triage system. Transferring this concept to oncology allows the navigator the opportunity to "triage" oncology patients not around success of therapy, but instead based on those committing to and completing oncology regimens. The resultant adherence possibly predicting better oncology disease-free outcomes.

The basis of this pilot study was to ask the navigators in the unit to colour code patients requiring primary chemotherapy into three groups. The colour coding was based on the well-known trauma coding score of Red (critical); yellow (could become critical); and green (not urgent). The navigators met with the treating oncology team; including the first contact physician post the MDM (Multidisciplinary Meeting). The physician informs the navigation team of his or her impression on the patient's reservations around primary chemotherapy. The physician coded the patient file based on feedback from the navigators. The navigator can change this code colour after each interaction with the patient.

The study was instituted to pilot if a colour code system would help the navigation team in identifying which patients may require more intensive navigation whilst undergoing NACT (neoadjuvant chemotherapy). The study was not to solve the many contributing factors to noncompliance and non-adherence but rather to provide a simple triage of files for the navigation team to better understand where additional services are required in order to ensure completion of NACT.

Successful multidisciplinary care involves not just the combination of choosing the correct treatment pathway for a patient but understanding the psycho-oncology factors that determine patient adherence/compliance with the recommended care pathways. The following factors were assessed as key to placing patients in different colour-coded pathways:

Patients coded green were found to have a fundamental belief in the medical system and were not swayed by external factors such as their social networks and the internet.

Patients in the yellow category had negative environmental associations with the concept of chemotherapy. These associations could have been formed either by the influence of friends and family or by the individuals own fear around chemotherapy. Patients who, during the therapy, had side effects to the treatment requiring admission or delays to further treatment were also placed in the yellow category.

Patients in the red category mostly started as not wanting to undergo primary chemotherapy due to intrinsic belief structures as to the damage chemotherapy would render to their physical being. Alternatively, some beliefs were set based on friends, family and the internet as to the harm of chemotherapy. Other patients within the red group had accepted alternative methods of treating cancer, predominantly sourced via the internet. 
Before colour coding the files in the unit, a trend as to which patients became "red code" was evident in certain traits such as: heightened anxiety levels around the concept of primary chemotherapy and desire to engage in alternative treatment strategies (with contact to the complementary oncology website noted). Financial concerns were not noted as reasons for being documented as a red, but rather placed patients in the yellow category; likewise, for treatment anxiety and logistical barriers. Age contributed as a reason for starting in the red category with elderly patients being far warier of starting with primary chemotherapy. Gajra et al. similarly observed that a lower preference for chemotherapy in geriatric patients was associated with lower quality of life, worse physical symptoms, self-function, and more side effects-related events in mid-treatment (11).

The colour code system provided navigators with an easy system for triaging patients and addressing issues of non-adherence and/ or non-compliance. Interventions to improve patient adherence included navigators rapidly assessing which patients required more telephonic interaction and implementation of visits in the form of a "meet and greet" system at the oncology unit before each chemotherapy session. A buddies network of community navigators (breast cancer survivors who are trained as lay navigators) was used to speak to patients about anticipated treatment regimens as well as managing fears of potential side effects. Health Education around both chemotherapy; anticipated side effects and understanding impact of chemotherapy on work and the home was provided. Further education was provided to those interested in only pursuing alternative treatment regimens. This was provided by a specialized navigator, trained in complementary medicine. Patients refusing chemotherapy was provided with regular ultrasound tumor assessment and specific counselling with the complementary health team and community navigators.

The majority of the code red patients who initially were against primary chemotherapy eventually underwent treatment and completed the course. The success of this pilot study suggests that targeted navigation file assessment system aids monitoring of patients on primary chemotherapy. This system provides the navigation team with an easy colour code to improve adherence or compliance in subsets of patients who initially refuse NACT or are battling with side effects on treatment. The focus of this study was not to analyze patient attitudes and behaviors pertaining to cancer treatment but rather to identify where more intensive navigation is required. The institution of systems that highlight potential non-adherence and non-compliance will facilitate studies on how to tackle the barriers to oncology care.

This study has now been registered for a prospective navigation study. Comparison studies using similar techniques in different units would quantify the benefit of implementing such colour coded navigation systems.

Ethics Committee Approval: Ethics committee approval was received for this study from the ethics committee of Pharma-Ethics(Pty) Ltd, Registration no. 99/13868/07. The ethics reference granted to us is: 170416525 .

Informed Consent: This a respective file analysis; that involves routine patient follow-up and communication by the navigators. Each patient at the time of their initial presentation to the unit signs a consent form allowing data file access. This form is attached to the ethics approval as mentioned above.

Peer-review: Externally peer-reviewed.

Author Contributions: Concept - C.A.V.; Design - C.A.B., D.vL.; Supervision - C.A.B., Y.R.; Resources - C.A.B., V.S.; Materials - C.A.B.; Data Collection and/or Processing - N.L.B., V.S.; Analysis and/or Interpretation - V.S., D.vL.; Literature Search - N.L.B., Y.R., B.vdB.; Writing Manuscript - C.A.B., Y.R., N.L.B., V.S.; Critical Review - C.A.B., B.vdB., T.vL; Other - V.S.

Acknowledgements: The authors would like to acknowledge the invaluable support that they have received from the navigation and research team in terms of conducting the study. This dedicated team have provided the unit with an opportunity to lay the groundwork for prospective work in the area of patient flow and service to our patients.

Conflict of Interest: The authors have no conflicts of interest to declare.

Financial Disclosure: The authors declared that this study has received no financial support.

\section{References}

1. National Health Laboratory Service. National Cancer Registry: Summary statistics, 2014. Johannesburg: National Health Laboratory Service; 2014.

2. Ferlay J, Colombet M, Soerjomataram I, Mathers C, Parkin DM, Pińeros M, Znaor A, Bray F. Estimating the global cancer incidence and mortality in 2018: GLOBOCAN sources and methods. Int J Cancer 2019; 144: 1941-1953. (PMID: 30350310). [Crossref]

3. Wright JC. Update on cancer chemotherapy: general considerations and breast cancer. Part II. J Natl Med Assoc 1985; 77: 691-703. (PMID: 3903172)

4. Goldhirsch A, Winer EP, Coates AS, Gelber RD, Piccart-Gebhart M, Thürlimann B, Senn HJ; Panel members. Personalizing the treatment of women with early breast cancer: highlights of the St Gallen International Expert Consensus on the Primary Therapy of Early Breast Cancer 2013. Ann Oncol 2013; 24: 220623. (PMID: 23917950)

5. Frenkel M. Homeopathy in cancer care. Altern Ther Health Med 2010; 16: 12-6. (PMID: 20486620)

6. Paltiel O, Avitzour M, Peretz T, Cherny N, Kaduri L, Pfeffer RM, Wagner N, Soskolne V. Determinants of the use of complementary therapies by patients with cancer. J Clin Oncol 2001; 19: 2439-2448. (PMID: 11331323) [Crossref]

7. Chawla N, Arora NK. Why do some patients prefer to leave decisions up to the doctor: lack of self-efficacy or a matter of trust? J Cancer Surviv 2013; 7: 592-601. (PMID: 23892559) [Crossref]

8. Robinson-White S, Conroy B, Slavish KH, Rosenzweig M. Patient navigation in breast cancer: a systematic review. Cancer Nurs 2010; 33: 127 140. (PMID: 20142736) [Crossref]

9. Chakrabarti S. What's in a name? Compliance, adherence and concordance in chronic psychiatric disorders. World J Psychiatry 2014; 4: 30-36. (PMID: 25019054) [Crossref]

10. Rayne S, Lince-Deroche N, Hendrickson C, Shearer K, Moyo F, Michelow P, Rubin G, Benn C, Firnhaber C. Characterizing breast conditions at an openaccess breast clinic in South Africa: a model that is more than cancer care for a resource-limited setting. BMC Health Serv Res 2017; 17: 63. (PMID: 28109290) [Crossref]

11. Gajra A, McCall L, Muss HB, Cohen HJ, Jatoi A, Ballman KV, Partridge AH, Sutton L, Parker BA, Magrinat G, Klepin HD, Lafky JM, Hurria A. The preference to receive chemotherapy and cancer-related outcomes in older adults with breast cancer CALGB 49907 (Alliance). J Geriatr Oncol 2018; 9: 221-227. (PMID: 29602735) [Crossref] 